\title{
Frontières
}

\section{Le long périple de la quête de transcendance de soi}

\section{Suzanne Hamel}

Volume 13, numéro 2, printemps 2001

Les morts de l'esprit

URI : https://id.erudit.org/iderudit/1074462ar

DOI : https://doi.org/10.7202/1074462ar

Aller au sommaire du numéro

Éditeur(s)

Université du Québec à Montréal

ISSN

1180-3479 (imprimé)

1916-0976 (numérique)

Découvrir la revue

Citer ce document

Hamel, S. (2001). Le long périple de la quête de transcendance de soi.

Frontières, 13(2), 76-80. https://doi.org/10.7202/1074462ar

\section{Résumé de l'article}

Par notre nature d'êtres humains, nous sommes riches d'une ressource innée dont la valeur est inestimable et souvent méconnue de nous, le Soi. C'est ce que la psychologie transpersonnelle fait ressortir. La condition essentielle pour " dé-couvrir » le Soi,- présent dans la profondeur de notre être -, est de mourir à l'ego. Dépasser les désirs et les peurs de l'ego permet de percevoir le monde à travers une conscience élargie et d'agir dans le monde avec compassion. L'article rapporte des attitudes propres à cette actualisation transcendante. Il s'agit des tout premiers résultats d'une étude présentement en cours qui vise à approfondir les attitudes favorisant l'actualisation du potentiel psychospirituel dans la vie quotidienne. 


\section{Résumé}

Par notre nature d'êtres humains, nous sommes riches d'une ressource innée dont la valeur est inestimable et souvent méconnue de nous, le Soi. C'est ce que la psychologie transpersonnelle fait ressortir. La condition essentielle pour " dé-couvrir » le Soi, - présent dans la profondeur de notre être -, est de mourir à l'ego. Dépasser les désirs et les peurs de l'ego permet de percevoir le monde à travers une conscience élargie et $d$ 'agir dans le monde avec compassion. L'article rapporte des attitudes propres à cette actualisation transcendante. II s'agit des tout premiers résultats d'une étude présentement en cours qui vise à approfondir les attitudes favorisant l'actualisation du potentiel psychospirituel dans la vie quotidienne.

Mots clés: actualisation transcendantepotentiel psychospirituel -

transpersonnel-Soi-ego-attitudes

\section{Abstract}

Through our nature as human beings, we are rich in an innate resource - the Self, which is invaluable and often unknown to us. This is what transpersonal psychology stresses. The necessary condition to "dis-cover" the Self, a condition present in the depth of our being, is dying with the ego. Surpassing the ego's desires and fears allows one to perceive the world through greater awareness and to act in the world compassionately. This article yields attitudes specific to this transcendental actualization. It contains all of the preliminary results of a currently underway study with the aim to heighten attitudes promoting the actualization of psycho-spiritual potential within everyday life.

Key words: transcendental actualization - psycho-spiritual potential-transpersonal-Self-egoattitudes

\section{Le long périple de la quête de transcendance de soi}

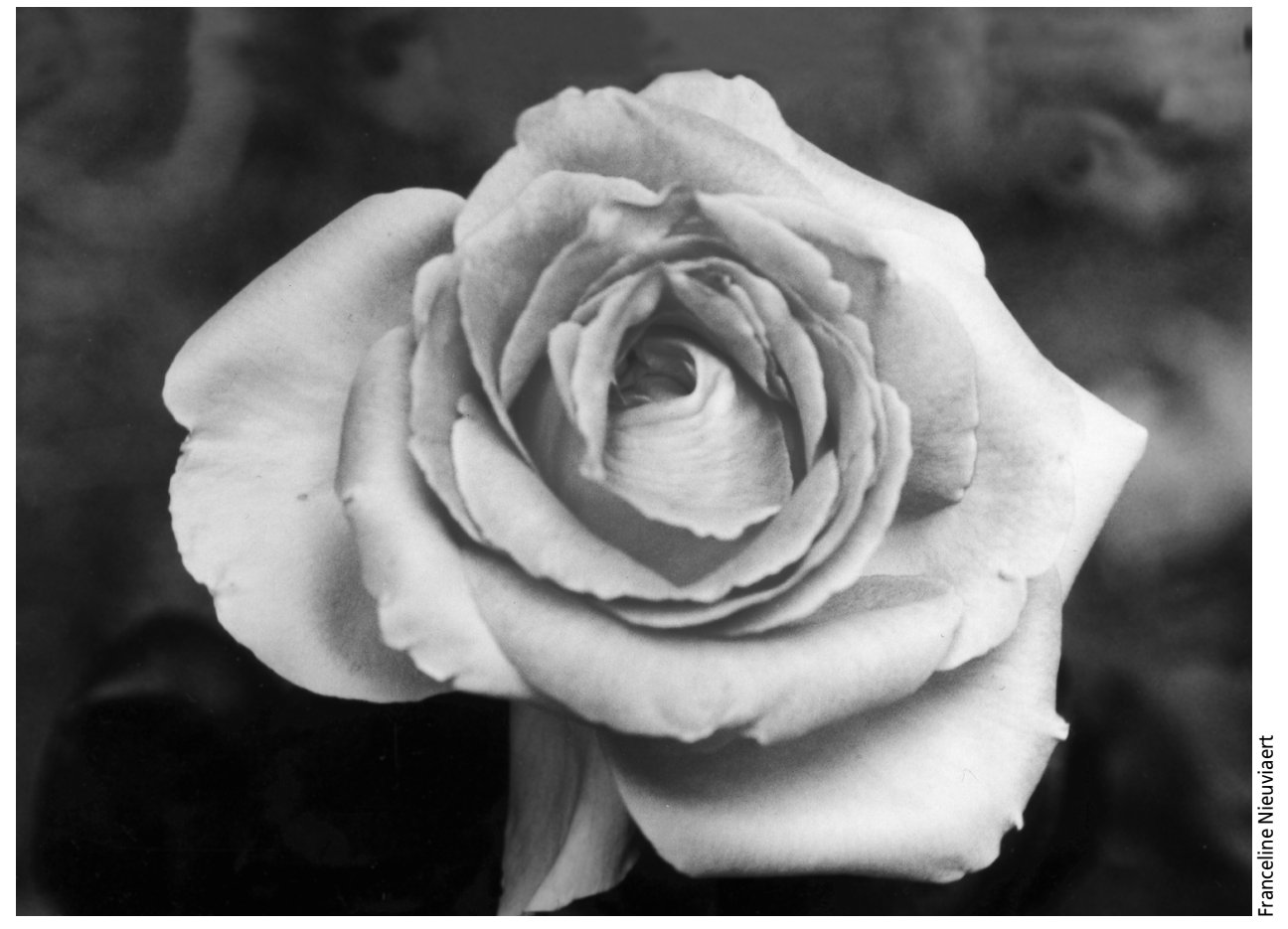

Suzanne Hamel, Ph.D.,

professeure adjointe au Département de psychologie de l'Université de Sherbrooke et chercheure

à l'Institut universitaire de gériatrie de Sherbrooke

De plus en plus, nous sommes conscients d'orienter notre vie en fonction des motivations qui nous animent. Pour toutes sortes de raisons, il est tentant, voire pressant, de nous modeler aux grandes tendances de notre culture sociale. Et cela, même quand on en déduit qu'elles sont déshumanisantes. Il n'est pas rare que nous nous laissions entraîner par nos motivations de protection, de sécurité ou d'expansion du moi à travers des conduites comme l'appât du gain, la compétition, la course à la performance, la réussite financière ou sociale. Or, pour vivre dans une société humanisante, nous sommes appelés à apaiser notre soif de satisfaire les désirs insatiables de notre ego afin de laisser émerger le Soi (ou le Moi véritable $)^{1}$. Nous sommes invités à la transcendance de soi, c'est-à-dire à satisfaire nos motivations d'ordre supérieur dans nos actions quotidiennes.

Pour vivre heureux et serein, nous devons abandonner notre esprit individualiste, celui qui naît de la centration sur nos préoccupations personnelles, pour nous 
identifier au Soi, ce centre permanent d'authenticité disponible en nous. Assagioli appelle l'Esprit cette source d'inspiration universelle et intrinsèque à toute personne qui accepte de percevoir et d'agir au-delà de son monde restreint:

L'Esprit constitue l'élément de transcendance, de supériorité, de permanence, de liberté, d'intériorité, de créativité, d'harmonie et de synthèse dans tout ce qui se manifeste, tant individuellement que collectivement. Ainsi, dans l'homme, est spirituel, à quelque degré, tout ce qui le pousse à transcender son exclusivisme égoïste, ses peurs, son inertie, son hédonisme, tout ce qui le porte à discipliner, à dompter, à diriger les forces désordonnées, instinctives et affectives, qui s'agitent en lui, tout ce qui le pousse à reconnaître une réalité plus large et plus haute, sociale ou idéale, et à s'insérer en elle en repoussant les limites de sa personnalité propre ${ }^{2}$.

Le présent article vise à illustrer - à l'aide d'un exemple tiré d'une recherche qualitative en cours sur l'actualisation du potentiel psychospirituel ${ }^{3}$ - des attitudes propres à la naissance transpersonnelle et à la satisfaction de motivations d'ordre supérieur.

\section{LA PSYCHOLOGIE TRANSPERSONNELLE}

Par notre nature humaine, nous sommes riches d'une ressource innée ${ }^{4}$ dont la valeur est inestimable et souvent méconnue de nous, le Soi. C'est ce que la psychologie transpersonnelle - cette approche qui est née de l'humanisme - met en relief. Le Soi se définit comme le noyau le plus authentique de nous-mêmes, c'est-à-dire dépouillé de nos peurs et de nos illusions. Il est intimement lié à ce que Jung et Assagioli ont appelé l'inconscient supérieur ou le supraconscient, c'est-à-dire la source de nos tendances les plus élevées sur le plan de la conscience, de la volonté et de l'amour altruistes $^{5}$. Le Soi est expérimenté comme le centre profond de notre être lié au sacré, au divin ou à l'Esprit. Toutefois, la condition essentielle pour « dé-couvrir » le Soi est de mourir à l'ego. Cela se produit chaque fois que nous renonçons à nos tendances égocentriques (certains auteurs diront égoïstes). Dépasser les désirs et les peurs de l'ego permet de percevoir le monde à travers une conscience élargie par le discernement et le détachement, et d'agir dans le monde avec créativité et compassion $^{6}$. Quand nous réussissons à dominer nos caprices, nos faux besoins, nos craintes irraisonnées, à garder une vie simple et à être plus présents aux autres, nous pouvons alors vivre des moments d'unification de

\section{UNIVERSELLE ET INTRINSÈQUE À TOUTE PERSONNE QUI ACCEPTE}

DE PERCEVOIR ET D'AGIR AU-DELÀ DE SON MONDE RESTREINT.

notre être à un niveau supérieur de conscience et d'amour ${ }^{7}$. Toutefois, on peut se demander comment relever un tel défi dans un monde qui valorise la réussite personnelle, la compétition et l'argent? Comment vivre le détachement et la compassion quand l'esprit de notre époque nous incite à la superficialité et à l'individualisme? Ces valeurs profondes qui prennent leur source dans notre Moi véritable (le Soi) ont peu de chances de s'actualiser quand l'ego prend toute la place.

L'ego constitue la partie instable de notre personnalité. Il est composé de nos peurs, nos désirs, nos émotions, nos perceptions. Tous sont susceptibles de changer au gré des événements, des modes, de la culture ${ }^{8}$. Il nous est difficile de nous départir de tous ces attachements mouvants sans «être une volonté $^{9} »$. Autrement dit, pour mourir à l'ego, il nous faut «vouloir» répondre à des motivations d'ordre supérieur. Cela est d'autant plus difficile que ces motivations ne nous apparaissent pas vraiment essentielles à notre survie, ni à notre bien-être matériel, personnel ou professionnel. C'est pourquoi la mort de l'ego représente d'abord et avant tout un acte de volonté qui se traduit par une ouverture à une dimension plus grande que nous-mêmes, une ouverture au sacré, au divin ${ }^{10}$. Parfois, cette ouverture est donnée sans que l'on ait à faire quoi que ce soit. C'est ce que l'on appelle une grâce ou un cadeau d'ordre surnaturel. Mais la plupart du temps, elle se réalise graduellement et de façon volontaire, à travers le long périple de la quête de transcendance de soi. Cette voie est celle d'où émerge l'Esprit, notre vrai moi capable de discernement, de détachement, de volonté créatrice et d'amour / compassion $^{11}$.

À partir d'une recherche qualitative en cours, et dans le but d'illustrer comment une personne peut volontairement se rapprocher du Soi dans la vie quotidienne, les attitudes transpersonnelles d'un sujet croyant et âgé de 80 ans ont été dégagées de son discours. Celui-ci a été choisi en fonction de son score relativement élevé au Profil d'actualisation transcendante $(\mathrm{PAT})^{12}$. Il s'agit d'un nouvel instrument ayant pour but d'estimer le niveau d'actualisation du potentiel transcendant ou psychospirituel. Il a été conçu pour des adultes de tout âge dont les personnes âgées peu scolarisées. Chacun des 30 items propose au répondant le choix entre trois attitudes illustrant chaque niveau de croissance: a) prépersonnel : une attitude visant principalement la conservation et la protection de la vie, b) personnel : une attitude visant principalement le développement de la personne et c) transpersonnel : une attitude visant principalement la transcendance de soi. À chaque item, le répondant choisit l'attitude qui lui est la plus familière. Après avoir rempli le questionnaire, il obtient un score global qui le situe dans la zone de croissance où il a plus souvent tendance à se réaliser. Plus le score est élevé, plus la personne a tendance à s'actualiser sur le plan psychospirituel.

En entrevue semi-dirigée, le sujet sélectionné a accepté de parler de ce qu'il considère le plus important dans sa vie. La méthode phénoménologique de Bachelor et Joshi a été utilisée pour l'analyse des données $^{13}$. Il s'agit d'une méthode d'analyse qualitative qui consiste d'abord à découper le discours du sujet en unités de sens naturelles après l'avoir transcrit. Ensuite, il faut éliminer les énoncés redondants afin de ne conserver que les thèmes centraux du discours, le plus souvent dans le langage exprimé par le sujet. Cette étape est suivie de celle où les thèmes centraux sont reformulés dans un langage plus professionnel, tout en demeurant le plus près possible du sens donné par le sujet. Et finalement, les thèmes centraux sont réorganisés de façon logique et cohérente afin de décrire le phénomène selon la perception du sujet. Cette description relative résume les éléments essentiels du discours de la personne sur le phénomène étudié. Cette synthèse lui est enfin soumise afin qu'elle confirme, infirme ou consolide ses propos. Cette dernière étape constitue une épreuve de validation de l'analyse du discours. La méthode phénoménologique comporte également une autre étape menant à une description générale quand il y a plusieurs sujets interrogés.

\section{DOUZE ATTITUDES TRANSPERSONNELLES RELEVÉES DANS LE TÉMOIGNAGE D'UNE PERSONNE ÂGÉE DE 80 ANS}

Nous présentons ici la description élaborée à partir du témoignage d'une seule personne âgée de 80 ans et croyante. Ses attitudes transpersonnelles, c'est-à-dire ses façons de penser et d'agir relatant son 
potentiel psychospirituel, ont été identifiées dans son discours en fonction des connaissances acquises sur l'actualisation transcendante, concept clé du cadre théorique transpersonnel ${ }^{14}$. Cette description des attitudes demeure relative car elle traduit l'expérience d'une seule personne. Toutefois, elle s'inscrit dans la réalisation d'une recherche en cours qui comprend une soixantaine d'entrevues et qui vise à élaborer une description générale des attitudes reliées au phénomène d'actualisation transcendante. Les attitudes transpersonnelles émergeant du discours de cette personne âgée prennent parfois la forme de sa réflexion sur la vie ou sur sa vie, alors qu'à d'autres moments, elles reflètent plutôt sa disposition intérieure ou ses comportements face aux événements.

\section{DÉCOUVRIR ET NOMMER \\ SA MISSION DE VIE \\ SUR LE PLAN SPIRITUEL}

Selon elle, lorsqu'on est jeune, pour être heureux, ça prend des buts. Maintenant, elle s'aperçoit qu'elle a fonctionné avec des buts partiels, et peu à peu, elle a découvert qu'il $\mathrm{y}$ avait une ligne de fond dans sa vie. Au début, celle-ci était difficile à nommer car il était plus facile pour elle de se fixer un but dans l'immédiat et de dire que plus tard elle aurait peut-être un autre but. Mais rendue à un âge avancé, elle reconnaît que ses buts temporaires convergeaient tous vers la même voie finalement. D'une certaine manière, elle considère que les êtres humains sont tous appelés à prendre la même route, et au fond, qu'ils n'arrivent jamais au bout de la route. L'important n'est pas de réaliser des buts spécifiques, mais plutôt de prendre plaisir à les réaliser. L'important est d'être en route et d'être toujours interpellée par quelque chose de plus grand qu'elle.

Être attentive et sentir l'appel de fond à l'intérieur d'elle lui a permis d'orienter sa vie, c'est-à-dire de découvrir sa vocation ou sa mission dans la vie. Selon elle, cet appel est présent depuis le très jeune âge. Il peut se traduire par le goût profond de s'occuper des personnes appauvries et des malades (valeur profonde : aider), ou encore, le goût d'être aviateur pour la Croix Rouge (valeur profonde : courage). C'est sentir que l'on porte en soi une orientation qui va nous donner telle ou telle ligne de vie significative.

\section{PRENDRE PLAISIR À RÉALISER
UN OBJECTIF DE DÉPASSEMENT UN OBJECTIF DE DÉPASSEMENT DE SOI}

Quand elle se fixe un objectif, par exemple, celui de grimper dans la montagne, il est certain qu'elle a du plaisir à atteindre son but. Mais les plaisirs ressentis à grimper dans la montagne, à découvrir peu à peu sa beauté et à dépasser sa fatigue font également partie du plaisir global. Avancer vers un objectif, c'est au fond réaliser quelque chose à laquelle elle tient sans toutefois en faire un absolu. Choisir un objectif de dépassement et prendre plaisir à toutes les étapes de sa réalisation la rendent heureuse. Par contre, si elle ne met pas les énergies pour le réaliser alors qu'elle se sent interpellée, elle ne se sent pas heureuse.

\section{SE TOURNER
VERS LES AUTRES}

La relation aux autres est importante pour le développement spirituel. Lorsqu'elle est centrée sur ses préoccupations, il arrive qu'elle pense beaucoup trop à elle et qu'elle tourne en rond. Se tourner vers les autres s'est révélé sa solution à la centration sur soi, mais ce n'est pas la seule. Elle sera d'une grande aide si elle est accompagnée de moyens comme la prière ou aller méditer en montagne.

\section{RECHERCHER \\ ET ACQUÉRIR
UNE LIBERTÉ REL \\ UNE LIBERTÉ RELATIONNELLE}

Rechercher et acquérir une liberté dans ses relations avec les autres lui permet toujours d'améliorer ses relations interpersonnelles. Il s'agit d'une liberté d'être. Autrement dit, c'est en devenant plus libre intérieurement qu'elle est plus libre pour établir des relations constructives. Et selon elle, la vraie liberté est fondamentalement liée à l'Esprit, au divin. Donc, elle ne peut être vraiment libre que dans l'application de valeurs profondes comme l'amour, la vérité ou la justice dans sa vie. Mais ça n'est jamais facile parce que la vie n'est jamais facile. L'important pour acquérir cette liberté est d'être en route.

Réussir à atteindre la vérité à l'intérieur d'elle, celle qui mène à la liberté, est l'apprentissage de toute sa vie dans la mesure où elle en fait une démarche de fond. Elle a dû faire le choix de cette forme de liberté et s'appliquer à la développer dans toutes ses relations. C'est une orientation qui lui fait vivre un profond bien-être quand elle est soutenue, mais aussi, des sentiments de regret quand elle s'en éloigne. Elle reconnaît que la liberté ne se réalise pas quand elle entretient des relations interpersonnelles troubles ou difficiles.

Elle dit avoir toujours besoin des autres, car dans toutes choses et dans toutes sciences, on a généralement besoin des autres. Malheureusement, elle remarque que les sciences, l'argent et la modernité sont des domaines très développés par les humains que nous sommes, mais que les sciences profondes du sens souffrent beaucoup du manque d'engagement.

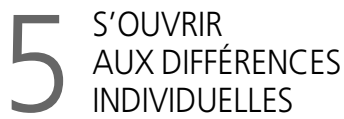

Tout se tient: la vie et les relations ne peuvent être séparées. Vivre avec les autres est difficile à cause des différences interpersonnelles qu'il lui faut dépasser. La qualité relationnelle dépend en partie des tempéraments des personnes en cause, selon que ceux-ci peuvent s'harmoniser ou non. D'après elle, les difficultés

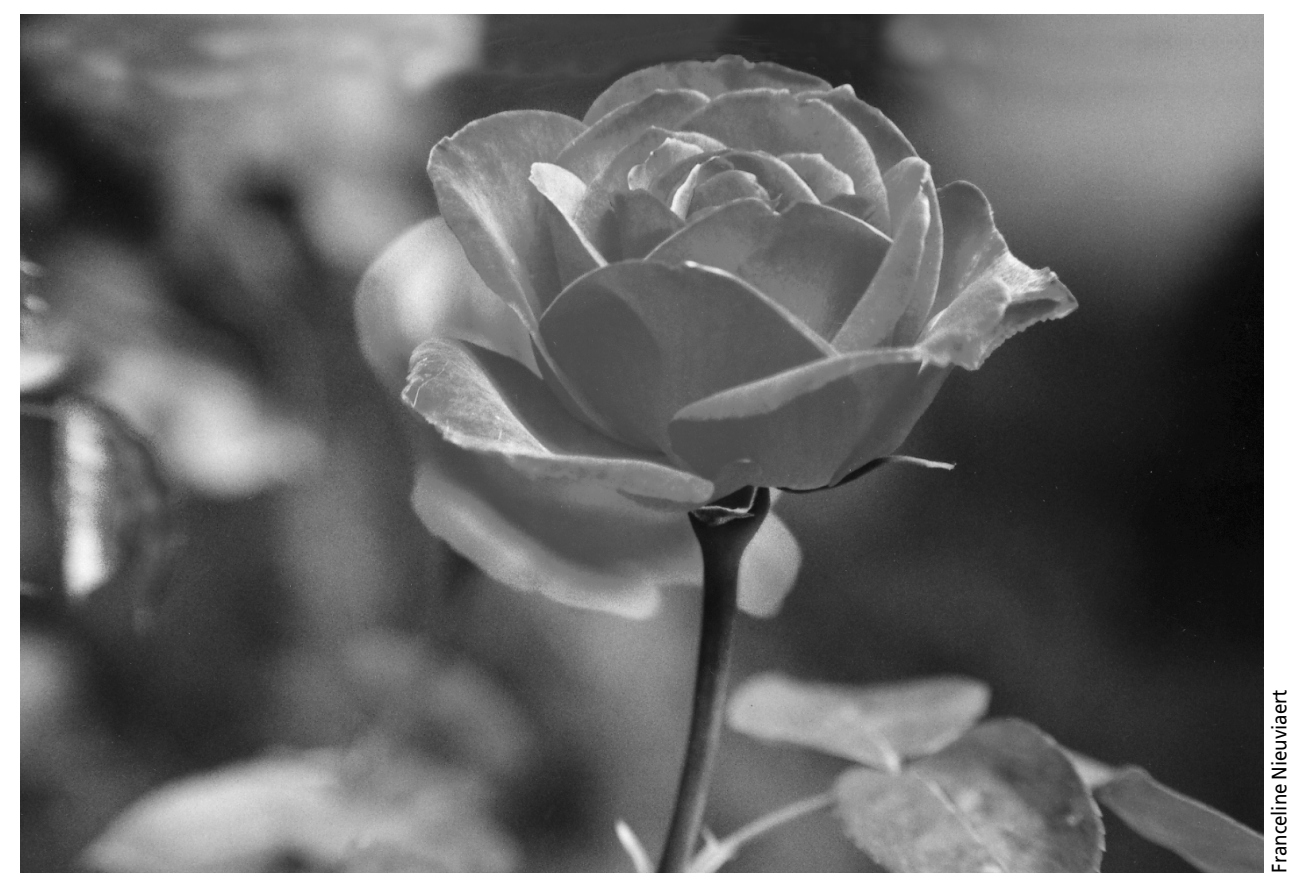


sont plus accentuées par le manque d'habilités à accepter et à gérer les différences individuelles.

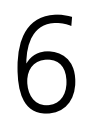

\section{PRENDRE LE TEMPS}

DE RÉFLÉCHIR

AVANT DE PARLER

Dans certaines situations conflictuelles, il peut être préférable de se taire. Avec l'âge, elle a appris que parler ou réagir n'arrange pas nécessairement les choses. Toutefois, les relations harmonieuses passent parfois par la nécessité de parler. Certaines personnes, ressentant un désir de paix dans leurs relations avec les autres, vont se taire alors qu'elles ressentent le besoin d'exprimer ce qui ne va pas. Comme elles ne savent pas comment faire quand elles se sentent attaquées et qu'elles ont peur de créer de la bagarre ou de faire éclater leur colère, elles préfèrent se taire, laisser aller les choses, considérer leur attitude soumise comme une de leurs faiblesses et penser que cela ne vaut pas la peine, que c'est inutile. Selon elle, pour pallier cette lacune relationnelle, il est bon de prendre un temps de recul pour réfléchir. Cela permet d'abord d'éviter les attitudes blâmantes parce que cela ne donne rien d'accuser l'autre. Ensuite, prendre le temps de réfléchir permet de trouver la façon adéquate d'agir. Prendre le temps de réfléchir a aussi pour conséquence que la parole mûrie sera mieux reçue par l'autre. Quand ces conditions sont présentes, reparler de la difficulté avec la personne visée est un atout pour améliorer la relation.

\section{CHOISIR ET LAISSER
LA LIBERTÉ DE CHOIX}

Toutes les techniques psychologiques modernes - comme celle où l'on apprend à parler au «je », c'est-à-dire à partir de son expérience et sans blâmer - sont aidantes pour améliorer les relations interpersonnelles. Toutefois, elle remarque que l'Évangile avait déjà tout dit sur la façon d'agir avec ses semblables. Dans cette perspective, elle observe que Jésus utilise un langage qu'on n'a pas l'habitude d'entendre. Jamais rien n'est imposé ou ordonné. La parole du Christ fait appel à la volonté et à la liberté de la personne. Par exemple, ce n'est jamais «Fais-ci, fais-ça, crois ceci, crois cela », mais plutôt « Si tu veux ».

\section{ACCEPTER DE FAIRE DES EFFORTS}

Dans toute chose, elle affirme qu'il y a des efforts à faire. C'est comparable à la montagne qu'elle grimpe : elle a chaud, elle sue, elle a soif, elle a l'impression de temps en temps de voir le sommet mais il est encore loin. Sa vie ressemble aux efforts en montagne. Quand elle était jeune, elle croyait arriver très vite au sommet de sa vie. Mais à 80 ans, elle constate qu'elle est encore loin du sommet. Par contre, elle sait aussi que c'est correct comme cela, que l'important est d'être en route.

Le mot «satisfaction» n'est pas approprié pour décrire le sentiment de liberté qui résulte de ses efforts. L'ouverture qui se crée quand elle est en route n'est pas de l'ordre de la satisfaction ordinaire comme celle d'avoir bien mangé. En fait, il n'y a pas de mots pour expliquer sa liberté intérieure qui grandit.

\section{ACCEPTER QUE DES ÉVÉNEMENTS DE VIE DIFFICILES SOIENT PRÉSENTS DANS SA VIE}

Les événements de vie difficiles à traverser l'ont fait passer à un autre registre de conscience. Par exemple, lors d'une maladie soudaine, elle a pris conscience de l'imminence de sa mort, et dans son expérience, elle a ressenti la sérénité du rapprochement avec le sacré, le divin. Par contre, quelques années plus tard, le même type d'événement éprouvant l'a amenée à des prises de conscience tourmentantes, comme celles de découvrir qu'elle n'acceptait pas de mourir, qu'elle supportait difficilement la souffrance et que sa foi en Dieu diminuait. Par conséquent, elle n'arrivait plus à prier, se concentrait sur sa souffrance et se sentait désespérément plus proche de la mort. Prendre conscience d'être si proche de la coupure de la vie terrestre par la mort, en avoir une vision plus radicale, lui a donné le vertige. Ce sentiment d'inconfort et de peur était d'autant plus fort car elle sentait sa foi profondément ébranlée. Cela lui a demandé une prière beaucoup plus dépouillée et intense.

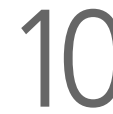

\section{SE LAISSER ALLER À DES SENTIMENTS DE RECONNAISSANCE ENVERS LA VIE}

Adoucir l'événement éprouvant par une vie spirituelle l'a amenée à se laisser aller à des sentiments de très grande reconnaissance envers la vie et ce qu'elle lui apprend. Même à un âge avancé, elle veut changer d'attitude dans sa façon de penser ou dans sa façon d'agir. Cela lui permet d'élargir sa conscience de la réalité et d'agir en conséquence. Par exemple, pour la première fois de sa vie à 80 ans, à la suite d'une grave maladie, elle fait du bénévolat auprès des personnes handicapées. Car elle réalise maintenant l'importance d'aider les gens à prendre conscience de tout ce qu'ils peuvent apprendre de leurs souffrances. Sa propre expérience de la souffrance, d'abord vécue dans la résistance et ensuite dans l'abandon spirituel, lui donne maintenant la certitude qu'à travers la douleur, qu'à travers quoi qu'elle vive, elle apprend finalement toujours quelque chose qui lui permet de mieux comprendre et d'aimer la vie.

\section{CONTEMPLER LABEAUTÉ SPIRTTUELLE OUAND ELLESE MANIFESTE DANS UNE AUTRE PERSONNE}

Vivre des moments de paix intérieure et d'harmonie avec elle-même, avec les autres et avec la vie prend aussi la forme de la contemplation, comme celle de contempler la beauté émanant de l'attitude unifiée d'une personne. Par exemple, elle est profondément touchée par les attitudes d'accueil aux autres, de dépassement de ses préoccupations, d'humilité et de simplicité d'une personne très malade qu'elle connaît. Elle vit cela comme des moments de grâce et d'émerveillement. Cette beauté est ressentie comme l'expression vivante du sacré et du divin. Souvent, elle a reconnu un trésor caché derrière les faits de la vie qui ne l'intéressaient pas au départ.

Elle a aussi découvert la beauté spirituelle dans les paroles de sagesse sortant naïvement de la bouche d'un enfant. Par exemple, le petit garçon qui avait répondu à la question d'un catéchète: "C'est qui Dieu? » Les gens avaient répondu de toutes sortes de manière, mais lui avait dit: « Dieu, c'est quand tu es bon. » Voilà une réponse qui pour elle n'a jamais fini d'être méditée. Découvrir ces perles qui se présentent par moments lui permet de reposer son être de ses vulnérabilités comme la colère ou l'orgueil et de lui donner un sentiment de paix et d'harmonie avec elle-même, les autres et la vie.

\section{SE DONNER DES MOYENS D'ÊTRE EN LIEN AVEC LE SOI}

\begin{abstract}
Prier
La prière est très importante pour elle, même si dans le quotidien, celle-ci n'est pas toujours régulière, ni parfaite. La prière est importante pour établir un lien intime avec Dieu. Cette relation n'est pas facile. Elle est même la relation la plus difficile parce que rechercher Dieu, c'est d'abord accepter de ne pas le voir. Il faut donc s'imprégner de la foi et de l'espérance. Prier, c'est d'abord se donner du temps. Elle découvre sans cesse la nécessité de l'arrêt. S'arrêter pour clarifier ce qu'elle vit, pour faire le point.
\end{abstract}


Lire des ouvrages philosophiques et spirituels

Elle s'est référée à des ouvrages philosophiques et spirituels dès le jeune âge. Cela s'est avéré une très bonne et solide source d'inspiration, même si elle ne saisissait pas très bien les écrits au début. Par exemple, les réflexions de Dürckheim ont été très ressourçantes.

\section{Identifier}

un symbole significatif

Identifier un symbole qui illustre l'image de la vie aide à être en route. Par exemple, elle a pris plaisir à grimper dans les montagnes, ce qui lui a permis de vivre un lien significatif avec la montagne et de l'utiliser comme symbole dans sa vie.

\section{Reconnaitre les facteurs aidants dans sa vie}

Il est important qu'elle reconnaisse ce qui l'aide dans sa vie. Par exemple, elle a beaucoup grimpé dans la montagne et, non seulement elle a découvert la beauté dans l'amont de la montagne, mais y a aussi découvert le silence, y a découvert Dieu, y a découvert Dieu dans le silence.

\section{Faire silence}

Pour prendre contact avec le sacré, elle affirme qu'il n'est pas absolument nécessaire d'avoir du silence autour de soi. Elle peut le découvrir également en parlant avec une autre personne. Cependant, elle est plus sûre de rester en lien avec le divin si elle crée un espace pour le silence intérieur, et cela, tout en étant en relation. Il arrive que l'intensité des émotions vécues devienne un obstacle à la découverte du silence. Par exemple, une valeur apparemment noble, comme celle d'être enragée de voir des injustices dans le monde, peut être un obstacle au silence. Par contre, passer à l'action concrète aide à s'en libérer. De fait, se sentir accrochée aux injustices peut être transmuté par le goût et l'action de vivre et d'aider les gens à vivre.

\section{UNE ATTITUDE TRANSPERSONNELLE VÉCUE AU QUOTIDIEN}

Ce témoignage nous a permis d'aborder une partie des attitudes permettant à la personne d'actualiser son potentiel psychospirituel. Mourir à l'ego, naître au Soi ou à l'Esprit et maintenir le plus souvent possible le lien avec le Soi (le véritable Moi) est en somme le défi de vivre le plus souvent possible dans la transcendance de soi. Il relève de l'ouverture psychologique et relationnelle, ensuite chapeautée par l'ouverture spirituelle, c'est-à-dire par l'appel du sacré et du divin dans toute réalisation et dans toute relation. Le Soi est infini, on ne l'atteint jamais une fois pour toutes. Comme l'a écrit Assagioli, le Soi est Pure Conscience et Amour. Il se manifeste parfois spontanément dans un mouvement descendant: l'Esprit se livre. Il s'agit de moments privilégiés de sacré, d'inspiration profonde et de grâce. Mais ces moments se manifesteront davantage s'il y a présence du mouvement ascendant, celui qui prend sa source dans la volonté de transcender l'ego. Chaque effort que nous déployons pour intégrer une attitude transpersonnelle dans nos vies permet d'élargir notre conscience des êtres et des événements et permet d'agir de façon plus appropriée, plus humaine, plus vraie et plus respectueuse du sacré et du divin dans la réalité quotidienne.

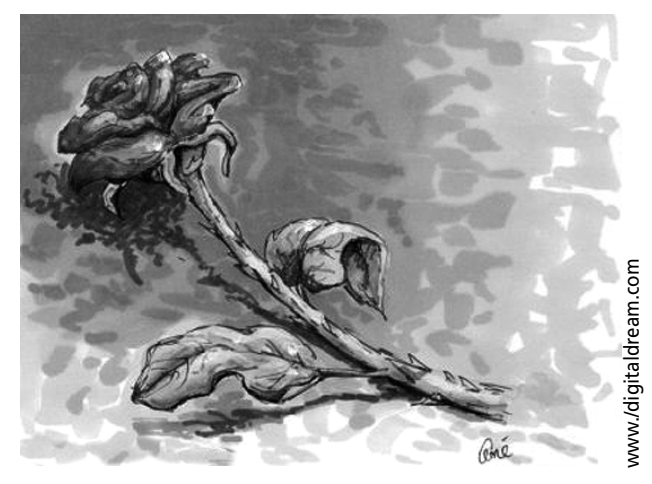

A. DESJARDINS, Les chemins de la Sagesse, Paris, La Table Ronde, 1972.

9 R. ASSAGIOLI, L'acte de volonté, Montréal, Centre de Psychosynthèse de Montréal, 1987.

10 A.H. MASLOW, The Farther Reaches of Human Nature, New York, Penguin Arkana, 1993.

$11 \mathrm{~S}$. HAMEL, «Étude exploratoire du besoin de transcendance et des modalités de satisfaction chez des jeunes adultes et des adultes étudiant en psychologie », Revue Interactions, vol. 1 , no 2, 1998, p. 292-318; S. HAMEL, R. LEFRANÇOIS et G. LECLERC, «La transcendance de soi : une tentative de définition", Revue québécoise de psychologie, vol. 20, no 3, 1999, p. 27-44.

12 S. HAMEL, G. LECLERC, R. LEFRANÇOIS et P. GAULIN, Manuel d'instruction et d'utilisation du Profil d'actualisation transcendante (PAT), Centre de recherche, Institut universitaire de gériatrie de Sherbrooke, Sherbrooke, Qc, Canada, 2001.

13 A. BACHELOR et P. JOSHI, La Méthode phénoménologique de recherche en psychologie, Québec, Les Presses de l'Université Laval, 1986.

14 A. H. MASLOW, ibid. note 4 ; V.E. FRANKL, Découvrir un sens à sa vie avec la logothérapie, Montréal, Éditions de l'Homme, 1988; R. ASSAGIOLI, ibid. note 2; K. WILBER, The Atman Project, A Transpersonal View of Human Development, Wheaton, Ill. U.S.A., Theosophical Pub. House, 1980; K.G. DÜRCKHEIM, La Voie de la transcendance, l'Homme à la recherche de son intégralité, France, Du Rocher, 1991. 